\title{
Dietary Patterns Related to Caries in a Low-Income Adult Population
}

\author{
Brian A. Burt ${ }^{\text {a }}$ Justine L. Kolker ${ }^{d}$ Anita M. Sandretto ${ }^{b}$ Ying Yuan $^{c}$ \\ Woosung Sohn ${ }^{d}$ Amid I. Ismail ${ }^{d}$ \\ Departments of a Epidemiology, ${ }^{b}$ Environmental Health Sciences, and 'Biostatistics, School of Public Health, \\ University of Michigan, and dDepartment of Cariology, Restorative Sciences and Endodontics, School of Dentistry, \\ University of Michigan, Ann Arbor, Mich., USA
}

\section{Key Words}

Dietary patterns $\cdot$ Soft drinks $\cdot$ Fat consumption $\cdot$ Plaque deposits $\cdot$ Oral hygiene $\cdot$ Low-income African-American households

\begin{abstract}
The aim of this study was to examine the relationship between dietary patterns and caries experience in a representative group of low-income African-American adults. Participants were residents of Detroit, Michigan, with household incomes below $250 \%$ of the federally-established poverty level $(n=1,021)$. Dietary histories were obtained by trained interviewers in face-to-face interviews with the adult participants, using the Block $98.2^{\circledR}$ food frequency questionnaire. Caries was measured by the ICDAS criteria (International Caries Detection and Assessment System). There were 200 dietary records whose data were judged to be invalid; these participants were omitted from the dietary analyses to leave $n=821$. Factor analysis identified patterns of liquid and solid food consumption, and the resulting factor scores were used as covariates in multivariable linear regression. Caries was extensive, with $82.3 \%$ of the 1,021 participants $(n=839)$ having at least one cavitated lesion. Nearly three quarters of the adult participants were overweight or obese. This population had severe caries, poor oral hygiene, and diets that are high in sugars and fats and low in fruits and vegetables. Apart from tap water, the most frequently consumed food
\end{abstract}

item by adults of all ages was soft drinks; $19 \%$ of all energy from sugar came from soft drinks alone. In both the bivariate analyses and in the regression model, frequency of soft drink consumption and the presence of gingival plaque deposits were significantly associated with caries. Interventions to promote oral health are unlikely to be successful without improvements in the social and physical environment.

Copyright $\odot 2006$ S. Karger AG, Basel

Sugars are an integral part of caries etiology [Gustafsson et al., 1954; Sheiham, 2001]. Even though the sugars/ caries relationship in the modern era of widespread fluoride exposure is not as clear and direct as was previously assumed [Burt and Pai, 2001], sugars' role in caries development is still not seriously disputed.

There is far less certainty, however, about the relationship between overall dietary patterns and caries, i.e. does a poor-quality diet predispose to caries? By the term 'poor-quality diet', we mean one that provides adequate energy but has an imbalance in nutrient intake when measured against recommended levels: too much fat and sugars and not enough fruits and vegetables. Such diets in high-income countries have been linked to a number of disease states [Dreeben, 2001; Kayrooz et al., 1998]. Poorquality diets have perhaps been linked most clearly to obesity, and the increase in obesity over recent years in the United States [Flegal et al., 2002] is a serious public health

\section{KARGER}

Fax +4161306 1234

E-Mail karger@karger.ch

www.karger.com
(C) 2006 S. Karger AG, Basel

0008-6568/06/0406-0473\$23.50/0

Accessible online at:

www.karger.com/cre
Dr. Brian A. Burt

Department of Epidemiology, School of Public Health, University of Michigan 109 Observatory Street

Ann Arbor, MI 48109-2029 (USA)

Tel. +1 734764 5478, Fax +1 734764 3192, E-Mail bburt@umich.edu 
problem. This is true for adults as well as for children, and obesity-related problems are found at all socioeconomic levels [Ogden et al., 2002]. From the dental viewpoint, we might intuitively expect that poor-quality diets promote caries, but apart from sugars' role there is little firm evidence to support such a relationship. Caries is certainly higher in children who have been high sucrose consumers all their lives when compared to children with lower lifetime sucrose intake [Ruottinen et al., 2004].

This paper examines the relationship between dietary patterns and caries experience in a representative group of low-income African-American adults in Detroit, Mich., USA. The study was conducted by the Detroit Center for Research in Oral Health Disparities. The Center has the overall goal of identifying reasons for the community-level and individual-level disparities in oral health found among this population, where families appear to live under much the same social and economic circumstances.

\section{Materials and Methods}

The study was conducted in accordance with the principles of the Declaration of Helsinki. All participants gave written consent to taking part. The study was approved by the Institutional Review Board for Health Sciences at the University of Michigan, Ann Arbor.

\section{Study Population}

The participants were African-Americans in Detroit with household incomes below $250 \%$ of the poverty level as defined by the federal government. Participants were selected using a multistage area probability sample design based on data from the US census of 2000. The 39 census tracts within Wayne County (where Detroit is located) with the highest proportion of low-income African-American households were selected, and a random sample of 565 blocks divided into 118 neighborhood segments was drawn from them. A household listing of these blocks was conducted from June to August 2002. From that household list, a number of housing units were chosen to yield the target sample of 1,000 persons for the first wave of data collection. Between September 2002 and August 2003, a field team visited the selected housing units and successfully screened and recruited 1,021 families to take part in the study. A family, for the purposes of this study, was defined as a primary caregiver and one African-American child who had not yet attained his/her 6th birthday.

\section{Data Collection}

Data were collected by interview and clinical dental examination at the same facility in Detroit. Questionnaires, completed by trained interviewers working with the adult participants, were designed to explore the sociodemographic and lifestyle characteristics of each adult and child, as well as their general and oral health history. Dietary histories were obtained by trained interviewers from adult participants, using the the Block $98.2{ }^{\circledR}$ food frequency questionnaire (FFQ) developed by Block Dietary Systems, Berkeley, Calif., USA. This FFQ asks the frequency of consumption of a wide variety of food items over the last year, plus the number of servings per ingestion and the portion size (judged by the respondent from photographs and food models of various portion sizes). Algorithms used by Block Dietary Systems, based on data from several National Food Consumption Surveys conducted by the US Department of Agriculture, permit the calculation of energy and nutrient intake as well as the frequency of consumption of food items.

\section{Measurement of Body Mass Index}

Participants were weighed on a calibrated digital scale and had their height measured from a wall-mounted tape measure, both from standard protocols. Body mass index (BMI), a broad measure of adiposity, is computed as weight in kilograms divided by the square of height in meters [National Institutes of Health, 2005]. Staffs were trained over a period of 1 month, and the quality of the interviews was regularly checked throughout the 11month data collection period by reviewing videotapes of the interviews. The investigators also conducted periodic face-to-face reviews with the interviewers.

\section{Measurement of Dental Caries and Plaque Deposits}

Caries was measured by the International Caries Detection and Assessment System (ICDAS) criteria applied by 4 trained dentists. ICDAS is a system of caries detection and assessment that was developed by an international group of caries researchers over the period 2002-2005 [Pitts, 2004]. It was intended that the criteria (a) reflect the current scientific understanding of the process of dental caries; (b) be adopted for use in dental surveillance, research, practice, and education, and (c) provide a foundation for incorporating and validating new caries diagnostic tools.

In the Detroit study, 4 dentists carried out the scoring for dental caries, periodontal conditions, and plaque deposits. Participants were examined in a standard dental operatory in the reclining position with a standard light source. The teeth were cleaned of loose debris with a scaler, where necessary, and plaque deposits assessed. Teeth were then examined for caries using the ICDAS criteria. Assessment of caries was primarily visual. Caries criteria necessarily varied slightly from pit and fissures to proximal to smooth surfaces. To illustrate the ICDAS criteria, the scoring system for pit and fissure caries was as follows:

1 Sound tooth surface: no loss of integrity after air-drying for $5 \mathrm{~s}$.

2 First visual change in enamel.

3 Distinct visual change in enamel.

4 Noncavitated surface with underlying dark shadow from dentine.

5 Localized enamel breakdown, with no dentine visible.

6 Distinct cavity with dentine visible.

7 Extensive distinct cavity with dentine visible.

The caries outcome measure in data analysis was $D_{1}$ MFS, where ' $D$ ' included both noncavitated and cavitated lesions, i.e. ICDAS categories 1-6 above. A second measure, $\mathrm{D}_{2} \mathrm{MFS}$, recorded just cavitated lesions (ICDAS categories 4-6). However, these two measures were tightly correlated (Pearson's $\mathrm{r}=0.95, \mathrm{p}<$ 0.001 ), so we used just $D_{1}$ MFS as the more complete outcome measure of caries. 
Plaque deposits were scored by the Patient Hygiene Performance (PHP) index [Podshadley and Haley, 1968] applied to the 'Ramfjord' index teeth [Ramfjord, 1959]. These teeth are the maxillary right first molar; maxillary left central incisor; maxillary left second premolar; mandibular left first molar; mandibular right central incisor, and mandibular right second premolar. The PHP index scores the degree of visible plaque deposits, after a disclosing rinse, as 0 (no plaque), 1 (recent plaque deposit), or 2 (long-term plaque deposit) in each of five zones of the tooth surface.

\section{Statistical Analyses}

Statistical analyses were conducted using software from SAS ${ }^{\circledR}$ [SAS Institute, 2005], and SUDAAN ${ }^{\circledR}$ [Research Triangle Institute, 2005] to estimate variances adjusted for the design effect. Significance was set at the conventional level of $\mathrm{p}<0.05$, and the Wald $\mathrm{F}$ test was used to derive $\mathrm{p}$ values.

Completed FFQs were mailed to Block Dietary Systems for analysis. All other data were entered twice using a custom-designed Access program. Data checking for error was conducted using SAS. Discrepancies between the first and second data entries were reviewed and resolved by a senior investigator (W.S.).

After data cleaning and filing, bivariate analyses were conducted to relate frequently reported foods to caries experience ( $D_{1}$ MFS scores). When the population was categorized into tertiles by $\mathrm{D}_{1}$ MFS scores, we selected the food items that were consumed at least once per day by at least $5 \%$ of the adult participants in the high-caries tertile, and again by at least $5 \%$ in the low-caries tertile. Some foods were reported in both tertiles, others were found only in one tertile and not the other. There were 29 such food items selected this way, and subsequent analysis was focused on them.

Factor analysis was conducted to identify the patterns of liquid and solid food consumption, and the resulting factor scores were then used as covariates in multivariable linear regression to relate dietary patterns to caries levels. Separate factor analyses were carried out for eight liquid food items (soft drinks were not included due to the high frequency of their consumption and strong bivariate association with caries) and 20 solid food items, using PROC FACTOR from the SAS package. The number of factors was determined by scree plots and by what made sense from the nutritional viewpoint. The resulting factor scores, along with the weekly servings of soft drinks, were then used as continuous independent variables in the multivariable linear regression model with $D_{1}$ MFS values as the continuous dependent variable. Square root transformation was performed to improve normality. The multivariable linear regression was carried out by using PROC REGRESS available in SUDAAN, incorporating sampling design and weights. The other independent variables included were: (a) oral health variables: the number of times teeth were brushed during the last week, and PHP plaque scores; (b) demographic variables: age, education, income, employment status.

We carried out backward elimination model selection to determine the most parsimonious predictive model for caries. We began with the full model that included all independent variables, proceeding at each step by dropping from the model the variable with the highest $\mathrm{p}$ value. (Demographic variables were retained because of their fundamental importance, regardless of their statistical significance.) This process was repeated until only food, drink, or oral hygiene variables with a $\mathrm{p}$ value $<0.10$ remained in the model.

Diet and Adult Caries

\section{Results}

The demographic characteristics and BMI of all adult participants who had at least one tooth $(\mathrm{n}=1,005)$, related to dental caries experience as measured by the $\mathrm{D}_{1}$ MFS index, are shown in table 1 . Socioeconomic status is not included because the study population, by design, was clustered at the lower end of the socioeconomic status spectrum.

\section{Nutrient Intake and BMI}

The study began with 1,021 adults. There were 200 FFQ interviews classed as invalid because they included far too many $(\geq 17)$ or far too few $(\leq 3)$ food items per day relative to national norms. Block Dietary Systems identified these records which were then eliminated from further dietary analyses. All analyses on diet and dietary patterns are therefore based on $n=821$, the completed FFQs considered valid. There were still some missing data in a few FFQs, which is why the totals in some analyses are less than 821 .

The BMI is a broad measure of weight related to height. From the data in table 1, it can be seen that nearly three quarters of the participants were either overweight or obese. Average daily energy intake for adult females was 2,090 kilocalories, which again is above average and consistent with the profile of overweight and obesity. The proportions of participants who were overweight or obese increased with increasing age: those aged under 26 were $68 \%$ overweight or obese, for those aged 26 to $<46$ years it was $74 \%$, and for those 46 or older it was $78 \%$. Adults ingested $13.5 \%$ of total energy from protein, $39.4 \%$ from fats, and $47.1 \%$ from carbohydrates (15.0\% from sugars).

Yet another indicator of poor overall dietary quality comes with the reported consumption of fruits and vegetables. Only 7.4\% reported eating two or more servings of vegetables per day and $7.8 \%$ two or more servings of fruit.

The most frequently reported food on a daily basis was tap water. The second most-reported food consumed daily was sugared soda (reported by $30.3 \%$ ), and the third was potato chips (22.1\%). Fried chicken was ranked ahead of green salad. Food sources for sugars for these adults were predominantly liquid. Soft drinks dominated, with $19 \%$ of energy from sugars coming from carbonated soft drinks alone.

\section{Caries Status and Plaque Deposits}

The overall weighted kappa coefficient for inter-examiner reliability in recording caries was 0.83 and the 
Table 1. Demographic characteristics and $\mathrm{BMI}^{1}$ related to caries experience $\left(\mathrm{D}_{1} \mathrm{MFS}\right)^{2}$ among participating adults with at least one tooth $(n=1,005)$ in phase 1 of data collection by the Detroit Center for Research on Oral Health Disparities

\begin{tabular}{|c|c|c|c|}
\hline Characteristics & Participants & $\begin{array}{l}\text { Weighted percent } \\
\pm \mathrm{SE}\end{array}$ & $\begin{array}{l}\text { Weighted mean } \\
\mathrm{D}_{1} \mathrm{MFS} \pm \mathrm{SE}\end{array}$ \\
\hline \multicolumn{4}{|l|}{ Sex } \\
\hline Male & 55 & $5.5 \pm 0.8$ & $41.2 \pm 3.9$ \\
\hline Female & 950 & $94.5 \pm 0.8$ & $47.8 \pm 1.3$ \\
\hline \multicolumn{4}{|l|}{ Age, years } \\
\hline $14-24$ & 342 & $34.0 \pm 1.8$ & $34.9 \pm 1.5$ \\
\hline $25-34$ & 464 & $46.2 \pm 2.1$ & $47.5 \pm 1.7$ \\
\hline $35-44$ & 143 & $14.2 \pm 1.0$ & $68.7 \pm 3.1$ \\
\hline $45-54$ & 42 & $4.2 \pm 0.5$ & $73.4 \pm 6.0$ \\
\hline $55+$ & 14 & $1.4 \pm 0.5$ & $98.6 \pm 10.9$ \\
\hline \multicolumn{4}{|l|}{ Education, years } \\
\hline 7-12 (less than high school) & 456 & $45.5 \pm 1.7$ & $46.9 \pm 1.7$ \\
\hline 13-14 (high school graduate) & 327 & $32.2 \pm 1.9$ & $47.2 \pm 1.8$ \\
\hline 15 (some college, no degree) & 194 & $19.5 \pm 1.9$ & $47.3 \pm 3.2$ \\
\hline 16-19 (associate degree or higher) & 28 & $2.8 \pm 0.7$ & $60.6 \pm 4.3$ \\
\hline \multicolumn{4}{|l|}{ Employment } \\
\hline Yes (full time or part time) & 389 & $38.7 \pm 2.3$ & $45.3 \pm 1.8$ \\
\hline No & 616 & $61.3 \pm 2.3$ & $48.9 \pm 1.5$ \\
\hline \multicolumn{4}{|l|}{ Family income, USD } \\
\hline$<10,000$ & 440 & $43.8 \pm 2.4$ & $47.1 \pm 1.9$ \\
\hline $10,000-19,000$ & 278 & $27.7 \pm 1.6$ & $48.3 \pm 2.3$ \\
\hline $20,000-29,000$ & 164 & $16.3 \pm 1.1$ & $46.2 \pm 2.5$ \\
\hline $30,000-39,000$ & 85 & $8.4 \pm 1.0$ & $45.3 \pm 2.7$ \\
\hline 40,000 or more & 38 & $3.8 \pm 0.7$ & $55.6 \pm 4.1$ \\
\hline \multicolumn{4}{|l|}{ CDC BMI level $^{1}$} \\
\hline Less than healthy $(\mathrm{BMI}<18.5)$ & 23 & $2.1 \pm 0.6$ & $54.2 \pm 36.4$ \\
\hline Healthy (BMI 18.5 to $<25$ ) & 263 & $26.3 \pm 1.7$ & $47.6 \pm 36.7$ \\
\hline Overweight (BMI 25 to <30) & 242 & $24.8 \pm 1.4$ & $49.5 \pm 46.8$ \\
\hline Obese (BMI 30+) & 477 & $46.8 \pm 1.5$ & $46.1 \pm 29.3$ \\
\hline
\end{tabular}

${ }^{1}$ Centers for Disease Control and Prevention, http://www.cdc.gov/nccdphp/dnpa/bmi/bmi-adult.htm; accessed June 9, 2005.

${ }^{2}$ Decayed, missing, and filled tooth surfaces in adults, ' $\mathrm{D}_{1}$ ' signifies noncavitated as well as cavitated carious lesions.

weighted kappa coefficient for intra-examiner reliability was 0.74. Caries was commonplace and severe in this population, with 839 of the 1,005 dentate participants having one or more untreated cavitated lesions. In those aged 14 to $<35$ years only $0.2 \%$ had no carious lesions. In those aged 35 to $<47(\mathrm{n}=140)$ it was $1.9 \%$, and in those aged 47 or more $(n=38)$ it was $3.6 \%$.

The only plaque measure significantly related to $D_{1}$ MFS scores was gingival plaque, i.e. visible plaque around the gingival margin after a disclosing rinse $(\mathrm{p}<$ 0.02). Total PHP scores, proximal surfaces and middle surface scores were not significantly related to caries experience.

\section{Diet and Caries}

For the independent variable in bivariate analyses, we used tertiles of the proportion of total energy (we called this energy \%) from the food item. By this measure $D_{1}$ MFS scores were not related to the intake of protein or fat, but they were significantly related to carbohydrate intake (table 2), despite the absence of a trend. Because carbohydrates include both non- and low-cariogenic starches 
Table 2. Mean $D_{1}$ MFS scores for adults $(n=821)$ by tertiles of the proportion of energy (energy \%) from carbohydrates

\begin{tabular}{|c|c|c|c|c|}
\hline Energy \% from all carbohydrates & Participants & $\begin{array}{l}\text { Mean } \\
\mathrm{D}_{1} \mathrm{MFS}\end{array}$ & $\begin{array}{l}\text { Standard } \\
\text { deviation }\end{array}$ & $\mathrm{p}$ \\
\hline Lowest tertile ( $\leq 44.1 \%$ energy from carbohydrate) & 272 & 45.6 & 28.5 & \multirow[t]{3}{*}{0.03} \\
\hline Middle tertile (44.1-50.4\% energy from carbohydrate) & 271 & 51.3 & 32.4 & \\
\hline Highest tertile ( $\geq 50.4 \%$ energy from carbohydrate) & 266 & 49.7 & 29.4 & \\
\hline
\end{tabular}

There were 12 participants with some missing data not included.

Table 3. Mean $D_{1}$ MFS scores for adults $(n=821)$ by tertiles of the proportion of energy (energy \%) from sugars

\begin{tabular}{|c|c|c|c|c|}
\hline Energy \% from sugars & Participants & $\begin{array}{l}\text { Mean } \\
\mathrm{D}_{1} \mathrm{MFS}\end{array}$ & $\begin{array}{l}\text { Standard } \\
\text { deviation }\end{array}$ & $\mathrm{p}$ \\
\hline Lowest tertile ( $\leq 9.6 \%$ energy from sugars) & 268 & 44.4 & 26.9 & \multirow[t]{3}{*}{0.001} \\
\hline Middle tertile (9.6-17.8\% energy from sugars) & 267 & 49.7 & 31.3 & \\
\hline Highest tertile ( $\geq 17.8 \%$ energy from sugars) & 274 & 52.4 & 31.7 & \\
\hline
\end{tabular}

There were 12 participants with some missing data not included.

Table 4. Mean gingival plaque scores for adults $(n=821)$ by tertiles of $D_{1}$ MFS scores

\begin{tabular}{lllll}
\hline Mean $\mathrm{D}_{1}$ MFS & Participants & $\begin{array}{l}\text { Mean gingival } \\
\text { plaque score }\end{array}$ & $\begin{array}{l}\text { Standard } \\
\text { deviation }\end{array}$ & $\mathrm{p}$ \\
\hline Lowest tertile $(<31)$ & 268 & 0.76 & 0.37 & 0.02 \\
Middle tertile $(31$ to $<53)$ & 261 & 0.79 & 0.37 & \\
Highest tertile $(53+)$ & 260 & 0.88 & 0.40 & \\
\hline
\end{tabular}

There were 32 participants with some missing data not included.
[Lingström et al., 2000] as well as cariogenic simple sugars, when the same analysis was restricted to sugars (all kinds) the sugars-caries relationship was even more significant (table 3). $D_{1}$ MFS scores were not related to total PHP scores, but they were related to gingival oral hygiene scores (table 4).

We also expressed the independent variable as daily servings of food items. No relation was found between $\mathrm{D}_{1}$ MFS scores and intakes of vegetables, fruit, grain, meat, dairy products, tomato juice, orange juice, other real fruit juices, drinks with added vitamin C, and milk. In bivariate analysis, there was at first only a weak relation between $\mathrm{D}_{1}$ MFS and soft drink consumption, but this relationship was age confounded. The younger adults drank more soft drinks and less coffee than the older adults, and when age-adjusted the $D_{1}$ MFS scores were significantly related to soft drink consumption $(\mathrm{p}<0.01$; data not tabulated).

Factor analysis resulted in four factors for 20 solid food items and four factors for eight liquid food items (soft drinks were not included). The four solid food factors represented fruits, chips/candy, breakfast foods (e.g. eggs, sausage), and cheese snacks, and the four liquid food factors represented real fruit juice, milk, fruit-flavored drinks (e.g. HiC, Sunny Delight), and coffee/tap water. These factor scores, along with the weekly servings of soft drinks, were then used as continuous independent variables in the multivariable linear regression with $D_{1}$ MFS 
Table 5. Full regression model for caries predictors $\left(\mathrm{R}^{2}=0.24\right)$

\begin{tabular}{lccr}
\hline Independent variables and effects & Beta coefficient & SE beta & $\mathrm{p}$ \\
\hline Intercept & 4.62 & 0.34 & $<0.01$ \\
Liquid factor 1 & -0.17 & 0.22 & 0.46 \\
Liquid factor 2 & 0.33 & 0.21 & 0.13 \\
Liquid factor 3 & -0.07 & 0.15 & 0.67 \\
Liquid factor 4 & 0.18 & 0.21 & 0.38 \\
Solid food factor 1 & -0.04 & 0.21 & 0.85 \\
Solid food factor 2 & 0.09 & 0.24 & 0.71 \\
Solid food factor 3 & -0.27 & 0.21 & 0.22 \\
Solid food factor 4 & 0.17 & 0.21 & 0.43 \\
Soft drinks & 0.02 & 0.01 & 0.02 \\
Education: not high school graduate & reference & - & - \\
Education: high school graduate & 0.02 & 0.16 & 0.92 \\
Education: some beyond high school & -0.01 & - & 0.97 \\
Annual income below USD 10,000 & reference & 0.21 & - \\
Annual income USD 10,000-19,999 & -0.06 & 0.22 & 0.78 \\
Annual income USD 20,000+ & -0.01 & - & 0.98 \\
Employment: yes & reference & 0.18 & - \\
Employment: no & 0.31 & - & 0.10 \\
Age group <26 years & reference & 0.24 & - \\
Age group 26-30 years & 0.54 & 0.20 & $<0.03$ \\
Age group 31+ years & 2.01 & 0.02 & 0.51 \\
No. times teeth brushed last week & 0.01 & 0.25 & $<0.01$ \\
Gingival plaque score & 1.02 & &
\end{tabular}

\begin{tabular}{llcc}
\hline Independent variables and effects & Beta coefficient & SE beta & $\mathrm{p}$ \\
\hline Intercept & 4.60 & 0.24 & $<0.01$ \\
Education: not high school graduate & 0.00 & 0.00 & - \\
Education: high school graduate & 0.13 & 0.15 & 0.39 \\
Education: some beyond high school & 0.10 & 0.22 & 0.66 \\
Annual income below USD 10,000 & 0.00 & 0.00 & - \\
Annual income USD 10,000-19,999 & -0.05 & 0.21 & 0.81 \\
Annual income USD 20,000+ & -0.04 & 0.22 & 0.87 \\
Employment: yes & 0.00 & 0.00 & - \\
Employment: no & 0.29 & 0.18 & 0.12 \\
Age group <26 years & 0.00 & 0.00 & - \\
Age group 26-30 years & 0.57 & 0.22 & 0.02 \\
Age group 31+ years & 2.10 & 0.20 & $<0.01$ \\
Gingival plaque score & 1.03 & 0.22 & $<0.01$ \\
Soft drinks & 0.02 & 0.01 & 0.01 \\
\hline
\end{tabular}

Table 6. Final regression model for caries predictors by backward elimination selection procedure $\left(\mathrm{R}^{2}=0.24\right)$ scores as the outcome. The full model is shown in table 5 . Age, frequency of soft drink consumption and presence of gingival plaque were significantly related to caries prevalence $(\mathrm{p}<0.05)$.

The final model, after the backward model selection procedure was carried out, is shown in table 6 . The independent variables retained in the final model (apart from the demographic variables) were frequency of soft drink consumption and presence of gingival plaque. Age was negatively associated with caries severity, and soft drink consumption and presence of gingival plaque were positively associated with caries severity. These results are similar to those from the full model. Possible two-way interactions were also explored based on the final model, but none of them were statistically significant. 


\section{Discussion}

Dietary patterns have been defined as multiple dietary attributes operationalized as a single exposure [Kant et al., 2004]. To measure dietary patterns in this study, we first considered using an existing index from the literature. However, while a number of indexes to measure dietary quality have been devised, none has emerged as more complete than the others [Kant, 1996]. We developed our own Recommended Food Index, based on US Department of Agriculture recommendations, but it resulted in very low scores with little variance between individual participants. This indicated a population with a homogenous diet and low consumption of healthy foods, so we did not use the Recommended Food Index in this analysis. The approach adopted was to use factor analysis, which in this instance detects dietary patterns that reduce the number of variables by finding factors that comprise correlated dietary items [Kant, 2004]. Factor analysis has been shown to be a valid measure of dietary patterns when the factor loadings are compared with the results of a 7-day food diary [Togo et al., 2003].

The data in table 1 are illustrative of the poor social conditions in this area. The vast majority of families have no male head of household, which in itself raises more social problems. The data also show that this is a severely overweight population. Having nearly three quarters of the predominantly female participants either overweight or obese is well above the national average [Flegal et al., 2002]. Dietary quality is poor by several measures. The proportion of energy from fats is above the level recommended by the US Department of Agriculture, while the carbohydrates and protein levels are near the bottom of the recommended range. Only $7.4 \%$ reported consuming two or more servings of vegetables per day, and $7.8 \%$ two or more servings of fruit. Current recommendations are to consume $2-2^{1 / 2}$ cups of fruit and vegetables per day [US Department of Agriculture, 2005]. An alternative set of dietary guidelines has been published from Harvard University. These differ from those put out by the US Department of Agriculture mostly in recommending that a higher proportion of energy come from fats, but only unsaturated fats, and less from carbohydrates [Harvard University, 2005]. The diet of the participants in our study is still poor under the Harvard guidelines.

Dietary patterns of African-American adults are a subject that has not received much research attention, but our results are similar to what little information has been published. One study among African-Americans in North Carolina, drawn from all socioeconomic levels, found that participants' consumption of fruits and vegetables was below the levels advised in national recommendations [Gary et al., 2004]. This pattern is even more pronounced in our Detroit study, where the study population, by definition, came from low socioeconomic levels and we did not see the contrasting dietary patterns that would be expected in a population drawn from all socioeconomic levels. It seemed to us that at least part of the reason is the poor availability of good-quality fruits and vegetables in sections of the city, with cultural and economic issues compounding this complex relationship.

The literature on the relationship between plaque deposits and caries is mixed. There are studies which conclude that plaque deposits (or more vaguely 'poor oral hygiene') are a risk factor for caries [Kleemola-Kujala and Rasanen, 1982; Mascarenhas, 1998], while others find no such association [Payette and Brodeur, 1992; Etty et al., 1994; Arrow, 1998]. Meticulous oral hygiene has been shown to reverse the caries process in accessible root lesions [Nyvad and Fejerskov, 1986]. The mixed literature is probably a result of studies' using inappropriate plaque measures (e.g. scoring plaque from tooth surfaces where caries is unlikely to be found), measuring caries only in the cavitated stage, and fluoride effects. In our study, overall PHP index scores were not related to caries levels, but the overall scores included plaque measures from areas of the tooth surface where caries was unlikely, e.g. the middle of the labial surface. When PHP scores were confined to gingival areas only, we found a strong relationship between plaque deposits and caries.

There was a time when cariogenic foods were thought of as being mostly solid foods, e.g. candy, chocolate, baked goods. The influential Vipehölm study [Gustaffson et al., 1954] in the 1950s emphasized the cariogenicity of 'sticky' foods and downplayed the importance of sugar in liquid form. More recently, however, it has been documented that sugared beverages are a major risk factor for caries development in children [Levy et al., 2003; Marshall et al., 2003]. Our data confirm these findings and demonstrate that in this population the same picture applies to adults. It is noted that $55 \%$ of the adults' energy from sugars comes from a few types of drinks, for children the equivalent figure is 54\% [Kolker et al., 2005]. This suggests similarities between the diets of the adults and the children.

It was this clear evidence that sugar sources in this population were predominantly liquid that led us to lift soft drinks out of the factor analysis and leave it as a separate covariable. Soft drinks retained a highly stable level of statistical significance throughout the backward elimination model selection; this stability would seem to emphasize 
their importance in the carious process. Other drinks in the ever-growing menu of available beverages, real fruit juices included, are sugary and hence are potentially cariogenic, but it is notable that all combinations of both liquids and solids identified by the factor analysis had dropped out of the final model while soft drinks remained. The model also retained its $\mathrm{R}^{2}$ value of 0.24 . The conclusion would seem to be that the quality of the overall diet by itself does not much influence caries in this population, but soft drink consumption in the presence of plaque retention around the gingival margins certainly does.

Our findings are that gingival plaque deposits and soft drinks are the major individual-level determinants of caries in this population. This leaves the door open for several intervention areas, i.e. improving both oral hygiene and dietary quality, specifically cutting back on soft drink consumption. However, achieving permanent beneficial change in individual behavior will remain a challenge in this population unless there are also structural changes in the community and in key aspects of its social functioning. We cannot expect people living in deep poverty, without prospects for improvement, and for whom violence is a part of everyday life, to successfully adopt the habits of healthy diets and careful oral hygiene unless their physical and social environment is able to provide a measure of hope and dignity.

\section{Acknowledgements}

This study was supported by grant number U-54 DE 14261 from the National Institute for Dental and Craniofacial Research.

\section{References}

Arrow P: Oral hygiene in the control of occlusal caries. Community Dent Oral Epidemiol 1998;26:324-330.

Burt BA, Pai S: Sugar consumption and caries risk: a systematic review. J Dent Educ 2001; 65:1017-1023.

Dreeben O: Health status of African Americans. J Health Social Policy 2001;14:1-17.

Etty EJ, Henneberke M, Gruythuysen RJ, Woltgens JH: Influence of oral hygiene on early enamel caries. Caries Res 1994;28:132-136.

- Flegal KM, Carroll MD, Ogden CL, Johnson CL: Prevalence and trends in obesity among US adults, 1999-2000. JAMA 2002;288:17231727.

- Gary TL, Baptiste-Roberts K, Gregg EW, Williams DE, Beckles GL, Miller EJ III, Engelau MM: Fruit, vegetable and fat intake in a population-based sample of African Americans. J Nat Med Assoc 2004;96:1599-1605.

-Gustaffson BE, Quensel CE, Lanke LS, Lundquist C, Grahnen H, Bonow BE, Krasse B: The Vipehölm dental caries study. The effect of different levels of carbohydrates intake on caries activity in 436 individuals observed for five years. Acta Odont Scand 1954;11: 232-364.

Harvard University, School of Public Health: The Nutrition Source, Knowledge of Healthy Eating. http://www.hsph.harvard.edu/nutritionsource/EDBH_update.html, accessed December 8, 2005.

Kant AK: Indexes of overall diet quality: a review. J Am Diet Assoc 1996;96:785-791.

Kant AK: Dietary patterns and health outcomes. J Am Diet Assoc 2004;104:615-635.

Kant AK, Graubard BI, Schatzkin A: Dietary patterns predict mortality in a national cohort: the National Health Interview Surveys, 1987 and 1992. J Nutr 2004;134:1793-1799.
Kayrooz K, Moy TF, Yanek LR, Becker DM: Dietary fat patterns in urban African American women. J Community Health 1998;23: 453-469.

Kleemola-Kujala E, Rasanen L: Relationship of oral hygiene and sugar consumption to risk of caries in children. Community Dent Oral Epidemiol 1982;10:224-233.

Kolker J, Burt BA, Sohn W, Ismail AI: Disparities in dental caries: are sugared beverages a factor (abstract)? Prog Annu Session Int Assoc Dent Res, Baltimore, 2005;242.

Levy SM, Warren JJ, Broffitt B, Hillis SL, Kanellis MJ: Fluoride, beverages and dental caries in the primary dentition. Caries Res 2003;37: 157-165.

Lingström P, van Houte J, Kashket S: Food starches and dental caries. Crit Rev Oral Biol Med 2000;11:366-380.

- Marshall TA, Levy SM, Broffitt B, Warren JJ, Eichenberger-Gilmore JM, Burns TL, Stumbo PJ: Dental caries and beverage consumption in young children. Pediatr 2003;112: 184-191.

Mascarenhas AK: Oral hygiene as a risk indicator of enamel and dentin caries. Community Dent Oral Epidemiol 1998;26:331-339.

National Institutes of Health: Guidelines on Overweight and Obesity: Electronic Textbook. http://www.nhlbi.nih.gov/guidelines/ obesity/e_txtbook/appndx/apndx5.htm, accessed December 13, 2005.

Nyvad B, Fejerskov O: Active root surface caries converted into inactive caries as a response to oral hygiene. Scand J Dent Res 1986;94: 281-284.

Ogden CL, Flegal KM, Carroll MD, Johnson CL: Prevalence and trends in overweight among US children and adolescents, 1999-2000. JAMA 2002;288:1728-1732.
Payette M, Brodeur JM: Comparison of dental caries and oral hygiene indices for 13- to 14 year-old Quebec children between 1977 and 1989-1990. J Can Dent Assoc 1992;58:921922, 926-929, 932-933.

Pitts N: ICDAS: An international system for caries detection and assessment being developed to facilitate caries epidemiology, research and appropriate clinical management. Community Dent Health 2004;21:193-198.

Podshadley AG, Haley JV: A method for evaluating oral hygiene performance. Public Health Rep 1968;83:259-264.

Ramfjord SP: Indices for prevalence and incidence of periodontal disease. J Periodontol 1959;30:51-59.

Research Triangle Institute: SUDAAN Users' Manual, release 9.01, SAS-callable version. Research Triangle Park, Research Triangle Institute, 2005

Ruottinen S, Karjalainen S, Pienihakkinen K, Lagstrom H, Niinikoski H, Salminen M, Ronnemaa T, Simell O: Sucrose intake since infancy and dental health in 10-year-old children. Caries Res 2004;38:142-148.

SAS Institute: SAS Release 9.0. Cary, SAS Institute, 2005.

Sheiham A: Dietary effects on dental diseases. Public Health Nutr 2001;4:569-591.

Togo P, Heitmann BL, Sorensen TI, Osler M: Consistency of food intake factors by different dietary assessment methods and population groups. Br J Nutr 2003;90:667-678.

US Department of Agriculture: Dietary guidelines for Americans 2005; Key Recommendations. http://www.health.gov/dietaryguidelines/dga2005/recommendations.htm, accessed July 5, 2005. 